\title{
Proposed revision of the family Secoviridae taxonomy to create three subgenera, "Satsumavirus", "Stramovirus" and "Cholivirus", in the genus Sadwavirus
}

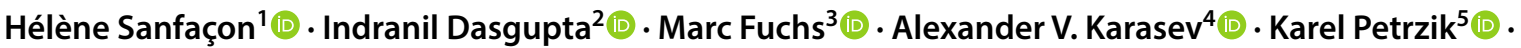

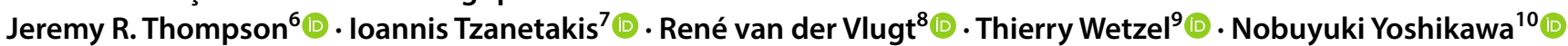

Published online: 17 December 2019

(c) Crown 2019

\begin{abstract}
We present a taxonomic proposal for revision of the family Secoviridae, a taxon of plant viruses in the order Picornavirales. We propose the reorganization of the genus Sadwavirus to create three new subgenera and to update the classification of five existing species. The proposed subgenera are "Satsumavirus" (one species: Satsuma dwarf virus), "Stramovirus" (two species: Strawberry mottle virus and Black raspberry necrosis virus) and "Cholivirus" (two species: Chocolate lily virus A and Dioscorea mosaic associated virus).
\end{abstract}

\section{Introduction}

The family Secoviridae was created in 2009 after amalgamation of the now-dissolved families Comoviridae and Sequiviridae and is the only family of plant viruses assigned to the order Picornavirales [13]. Members of the family, referred to as secovirids, share common properties with other members of the order: (1) a positive-strand RNA genome, which can be monopartite or bipartite, with each RNA linked at its 5' end to a small viral protein termed "VPg", (2) the production of large polyproteins that are cleaved by viral proteases, (3) a conserved "replication block" within the RNA1-encoded polyprotein that includes a type III helicase, the VPg, a cysteine protease related to the picornavirus $3 \mathrm{C}$ protease, and a type I RNA-dependent RNA polymerase, (4) a 25- to 30-nm icosahedral capsid with a pseudo $\mathrm{T}=3$ symmetry. The capsid is composed of 60 coat protein (CP) subunits, each consisting of three $\beta$-barrel domains that can be divided into a single large $\mathrm{CP}$, or into two or three smaller CPs depending on the genus.

Currently, there are eight genera in the family Secoviridae: the genera Sadwavirus, Torradovirus, Cheravirus, Sequivirus and Waikavirus, which do not belong to a subfamily, and three genera (Comovirus, Fabavirus, Nepovirus)
Handling Editor: Sead Sabanadzovic.

Hélène Sanfaçon

helene.sanfacon@canada.ca

1 Summerland Research and Development Centre, Agriculture and Agri-Food Canada, P.O. Box 5000, 4200 Highway 97, Summerland, BC VOH 1Z0, Canada

2 Department of Plant Molecular Biology, University of Delhi South Campus, New Delhi 110021, India

3 School of Integrative Plant Science, Cornell University, New York State Agricultural Experiment Station, Geneva, NY 14456, USA

4 Department of EPNN, University of Idaho, Moscow, ID 83844-2329, USA
5 Department of Plant Virology, Institute of Plant Molecular Biology, Biology Centre AS CR, Branisovska 31, 37005 Ceske Budejovice, Czech Republic

6 School of Integrative Plant Science, Cornell University, Ithaca, NY 14853, USA

7 Department of Entomology and Plant Pathology, Division of Agriculture, University of Arkansas, Fayetteville, AR 72701, USA

8 Wageningen University and Research, Droevendaalsesteeg 1, Wageningen 6708 PB, The Netherlands

9 DLR Rheinpfalz-Institute of Plant Protection, Breitenweg 71, 67435 Neustadt an der Weinstrasse, Germany

10 Plant Pathology Lab, Faculty of Agriculture, Iwate University, Ueda 3-18-8, Morioka 020-8550, Japan 


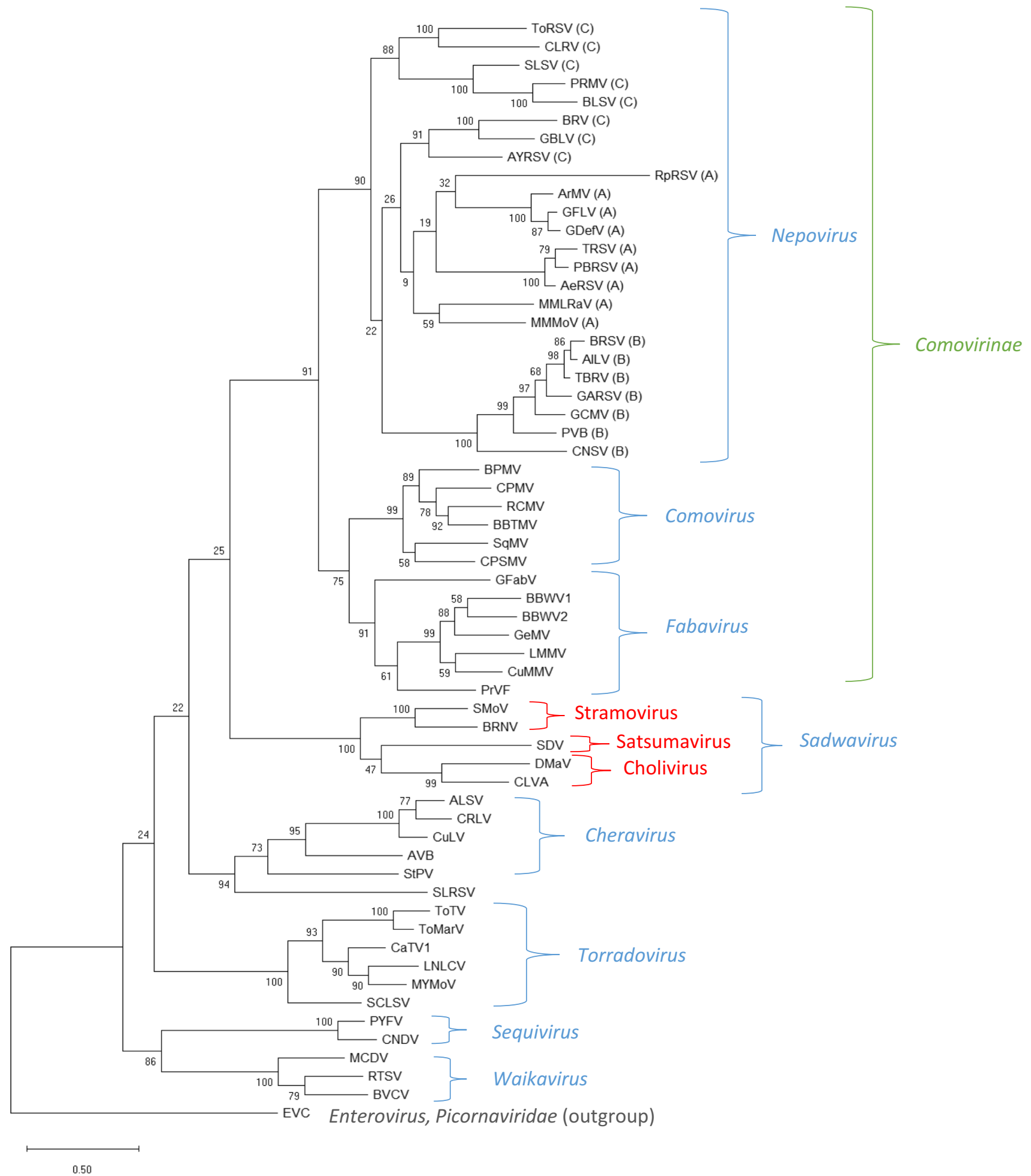

in the subfamily Comovirinae [16]. Within the family, phylogenetic relationships have been used to distinguish genera and subfamilies. In particular, the so-called "ProPol" sequence, which is defined as the sequence between the conserved catalytic cysteine (or serine) of the 3C-like protease and the GDD motif of the RNA-dependent RNA polymerase, has been used to refine the taxonomy of the family Secoviridae and its relationship to other members of the order [7, 8, 13]. Additional criteria have also been used to distinguish genera in the family: (1) the number of genomic RNAs (one for sequiviruses and waikaviruses, two for all other genera), (2) the number of protein domains and/ 
4Fig. 1 Maximum-likelihood phylogenetic analysis of the Pro-Pol amino acid sequence of type isolates from recognized species in the family Secoviridae. The alignment was generated using ClustalW in MEGA X, the tree was also built in MEGA X. Bootstrap values for each node are shown (1000 replicates). For each Secoviridae species, the Pro-Pol amino acid sequence was deduced from the nucleotide sequence of the corresponding genomic RNA from the type isolate. Abbreviations and sequence accession numbers are as follows: ToRSV (tomato ringspot virus, L19655), CLRV (cherry leaf roll virus, FR851461), SLSV (soybean latent spherical virus, KX424571), PRMV (peach rosette mosaic virus, KY646466), BLSV (blueberry latent spherical virus, AB649296), BRV (blackcurrant reversion virus, AF368272), GBLV (grapevine Bulgarian latent virus, FN691934), AYRSV (artichoke yellow ringspot virus, AM087671), RpRSV (raspberry ringspot virus, AY303787), ArMV (arabis mosaic virus, AY303786), GFLV (grapevine fanleaf virus, D00915), GDeF (grapevine deformation virus, HE613269), TRSV (tobacco ringspot virus, U50869), PBRSV (potato black ringspot virus, KC832887), AeRSV (aeonium ringspot virus, JX304792), MMLRaV (mulberry mosaic leaf roll associated virus, KC904083), MMMoV (melon mild mottle virus, AB518485), BRSV (beet ringspot virus, D00322), AILV (artichoke Italian latent virus, LT608395), TBRV (tomato black ring virus, AY157993), GARSV (grapevine Anatolian ringspot virus, HE774604), GCMV (grapevine chrome mosaic virus, X15346), PVB (potato virus B, KX656670), CNSV (cycas necrotic stunt virus, AB073147), BPMV (bean pod mottle virus, M62738), CPMV (cowpea mosaic virus, X00206), RCMV (red clover mottle virus, X64886), BBTMV (broad bean true mosaic virus, GU810903), SqMV (squash mosaic virus, AB054688), CPSMV (cowpea severe mosaic virus, M83830), GFabV (grapevine fabavirus, KX241482), BBWV1 (broad bean wilt virus 1, AB084450), BBWV2 (broad bean wilt virus 2, AF225953), GeMV (gentian mosaic virus, AB084452), LMMV (lamium mild mosaic virus, KC595304), CuMMV (cucurbit mild mosaic virus, EU881936), PrVF (prunus virus F, KX269865), SMoV (strawberry mottle virus, AJ311875), BRNV (black raspberry necrosis virus, DQ344639), SDV (satsuma dwarf virus, AB009958), DMaV (dioscorea mosaic associated virus, KU215538), CLVA (chocolate lily virus A, JN052073), ALSV (apple latent spherical virus, AB030940), CRLV (cherry rasp leaf virus, AJ621357), CuLV (currant latent virus, KT692952), AVB (arracacha virus B, JQ437415), StPV (stocky prune virus, DQ143874), SLRSV (strawberry latent ringspot virus, AY860978), ToTV (tomato torrado virus, DQ388879), ToMarV (tomato marchitez virus, EF681764), CaTV1 (carrot torradovirus 1, KF533719), LNLCV (lettuce necrotic leaf curl virus, KC855266), MYMoV (motherwort yellow mottle virus, KM229700), SCLSV (squash chlorotic leaf spot virus, KU052530), PYFV (parsnip yellow fleck virus, D14066), CNDV (carrot necrotic dieback virus, EU980442), MCDV (maize chlorotic dwarf virus, U67839), RTSV (rice tungro spherical virus, M95497), BVCV (bellflower vein chlorosis virus, KT238881). The Pro-Pol sequence of poliovirus (EVC, species Enterovirus C, NP_041277, genus Enterovirus, family Picornaviridae) was used as an outgroup to root the tree. Higher taxa are indicated with the following code: the three proposed subgenera in red, the genera in blue and the subfamily in green. In the case of nepoviruses, the letter in parentheses after each virus abbreviation indicates the subgroup

or processing sites within the polyproteins, (3) the number of CPs (one CP of 53-60 kDa for nepoviruses; two CPs of $35-40 \mathrm{kDa}$ and 20-25 kDa for comoviruses, fabaviruses and sadwaviruses; and three CPs of 20-25 kDa for cheraviruses, torradoviruses, sequiviruses and waikaviruses), (4) the presence of additional open reading frames (ORFs), e.g., one additional ORF on the RNA2 of torradoviruses in addition to the large polyprotein, and (5) the presence of subgenomic RNAs. Not all criteria need to be met simultaneously.

The genus Sadwavirus was created in 2004 [7] and originally included the species Satsuma dwarf virus, Strawberry latent ringspot virus and Strawberry mottle virus in order to classify isolates of homonymous viruses (abbreviations: SDV, SLRSV and SMoV). At the time, few secovirid sequences were available and phylogenetic relationships could not be clearly established. SDV and SLRSV shared the common property of having two CPs, whereas the number of CPs of SMoV isolates had not been experimentally determined, owing to difficulties with purifying this virus [15]. In 2009, SLRSV and SMoV were demoted from the genus [13]. SLRSV does not branch together with SDV in the ProPol phylogenetic tree. Rather it is related, albeit distantly, to cheraviruses [7] (see Fig. 1). SMoV is related to SDV in the Pro-Pol tree, but the number of protein domains in the RNA2 polyprotein was unclear, in particular the number of CPs.

New viruses with phylogenetic relationships to SDV have been described more recently (see Fig. 1). Black raspberry necrosis virus (BRNV) was found to be closely related to SMoV [5]. The number of protein domains in the RNA2 polyprotein of $\mathrm{BRNV}$, which includes the $\mathrm{CP}(\mathrm{s})$, has also not been determined experimentally. The species Black raspberry necrosis virus was created in 2009 and was classified in the family Secoviridae but was not assigned to a genus [13]. In 2016 and 2017, two new viruses, chocolate lily virus $\mathrm{A}$ (CLVA) and dioscorea mosaic associated virus (DMaV) were classified in additional unassigned species in the family: Chocolate lily virus A and Dioscorea mosaic associated virus $[1,2,6,19]$.

Recent analysis of cleavage sites in the polyproteins of SMoV revealed that this virus encodes two proteases: the RNA1-encoded 3C-like protease, which is common to all viruses in the family, a characteristic also shared with other members of the order, and the RNA2-encoded glutamic protease, which corresponds to a novel type of viral protease [9, $11,12]$. The RNA1 protease cleaves the RNA1 polyprotein at five sites, defining six domains in the polyprotein (Fig. 2). The presence of two protein domains upstream of the nucleotide-binding protein (NTB, putative helicase) domain is a feature shared with nepoviruses [4, 17, 18]. Examination of the polyproteins of BRNV, CLVA, DMaV and SDV revealed the presence of putative cleavage sites at similar positions that would imply a similar organization of the RNA1 polyprotein $[9,10]$ (Fig. 2). The RNA2 polyprotein 

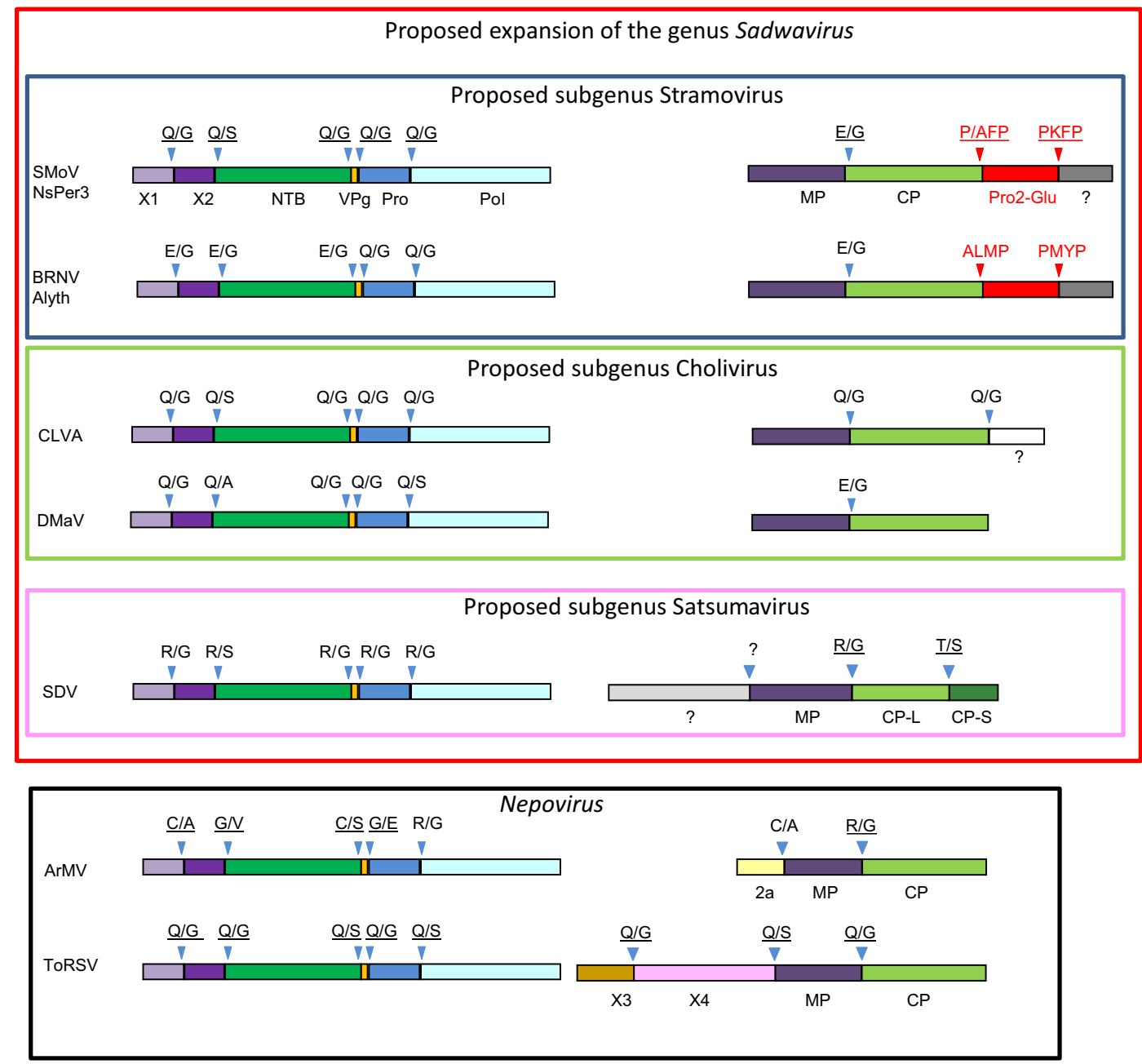

Fig. 2 Protein domains and protease cleavage sites in the polyproteins of members of the proposed expanded genus Sadwavirus. Cleavage sites that were confirmed experimentally by Edman degradation sequencing or by mutagenesis are underlined. Other cleavage sites were deduced based on amino acid sequence alignments of the entire RNA1 or RNA2 polyproteins. The genome organization of two representative members of the genus Nepovirus is shown for comparison. Cleavage sites recognized by the RNA1-encoded 3C-like protease

of a Canadian isolate of SMoV (isolate NsPer3) was cleaved at one site (between the MP and CP domains) by the RNA1encoded protease [9]. It was also cleaved at two sites by the RNA2-encoded glutamic protease (Fig. 2) [11]. Sequence comparison of SMoV and BRNV implied a similar genomic organization for BRNV (Fig. 2) [11]. These results strongly suggest that $\mathrm{SMoV}$ and BRNV have unique characteristics that distinguish them from SDV: (1) they encode one large are shown in black (and blue arrowheads). Cleavage sites recognized by the RNA2-encoded glutamic protease are shown in red (and red arrowheads). For strawberry mottle virus (SMoV), the genomic organization of Canadian isolate SMoV-NsPer3 is shown. The German isolate SMoV-1134 differs in that it is missing the last protein domain of the RNA2 polyprotein, located after the glutamic protease domain $[3,9,11,15]$. Abbreviations of virus names are as in Fig. 1

$\mathrm{CP}$ rather than two CPs; (2) the RNA2 polyprotein possesses additional protein domains downstream of the $\mathrm{CP}$ domain, including a novel glutamic protease domain. The presence of protein domains downstream of the $\mathrm{CP}$ is a new feature in the family Secoviridae. Signature sequences for the glutamic protease domain were not found in the RNA2 polyprotein of CLVA and DMaV [11]. Prediction of cleavage sites recognized by the RNA1-encoded 3C-like protease suggested the 
presence of a single large CP for CLVA and DMaV $[9,10]$, although this needs to be confirmed experimentally.

It was initially suggested that the identification of a novel protease domain in the RNA2 polyprotein of SMoV could justify the creation of a new genus to encompass BRNV and SMoV [11]. However, discussion within the ICTV Secoviridae Study Group (i.e., the authors of this manuscript) highlighted that phylogenetic relationships among BRNV, SMoV, SDV, CLVA and DMaV should also be recognized. Indeed, recent reexamination of phylogenetic relationships using the deduced amino acid sequence of the Pro-Pol region of type isolates for recognized species in the family confirmed that SDV, SMoV, BRNV, CLVA and DMaV group together as a single branch with a high degree of confidence (Fig. 1). In addition, phylogenetic trees based on the CP sequence (which is defined by the mapping of the cleavage sites as shown in Fig. 2) also suggested a common lineage for SDV, SMoV, BRNV, CLVA and DMaV, although the bootstrap analysis revealed a lower degree of confidence in the branching than with the Pro-Pol tree (Fig. 3). Thus, it was agreed that the corresponding five species should be classified in a common genus (Sadwavirus).

With the recent approval of additional taxonomic ranks including subgenera [14], we propose to create three subgenera in the genus Sadwavirus to classify these five viral species based upon the differences in the genomic organization of RNA2, including the presence (or absence) of the glutamic protease. The corresponding taxonomy proposal (2019.008P.N.v1.Sadwavirus_3subg) was conditionally approved by the ICTV executive committee and is awaiting ratification by the ICTV membership. Below is a summary of the taxonomic updates:
(1) Reorganization of the genus Sadwavirus to include three subgenera (proposed subgenera "Satsumavirus", "Stramovirus" and "Cholivirus") to classify five species (Satsuma dwarf virus, Strawberry mottle virus, Black raspberry necrosis virus, Chocolate lily virus A and Dioscorea mosaic associated virus), with Satsuma $d$ warf virus remaining the type species. Common characteristics of members of the genus Sadwavirus: group as a single lineage in both the Pro-Pol and CP trees, six protein domains in the RNA1 polyprotein. The name Sadwavirus is kept for the genus. Since Strawberry mottle virus was assigned to Sadwavirus between 2004 and 2009, this will prevent confusion.

(2) Creation of the subgenus "Satsumavirus", with Satsuma dwarf virus as the only species. Characteristics of members of the subgenus: one viral protease (the RNA1-encoded cysteine protease), two CPs, group as a single sub-branch in the Pro-Pol and CP trees.

(3) Creation of the subgenus "Stramovirus", encompassing Strawberry mottle virus and Black raspberry necrosis virus (proposed name derived from strawberry mottle virus, the best-characterized virus). Characteristics of members of the subgenus: two viral proteases (the RNA1-encoded 3C-like cysteine protease and the RNA2-encoded glutamic protease), a single large CP, group as a single sub-branch in the Pro-Pol and CP trees.

(4) Creation of the subgenus "Cholivirus", encompassing Chocolate lily virus A and Dioscorea mosaic associated virus (proposed name derived from chocolate lily virus $\mathrm{A}$, the first characterized member). Characteristics of members of the subgenus: one viral protease (the RNA1-encoded cysteine protease), probably a single large CP (based on putative cleavage sites), group as a single sub-branch in the Pro-Pol and CP trees. 


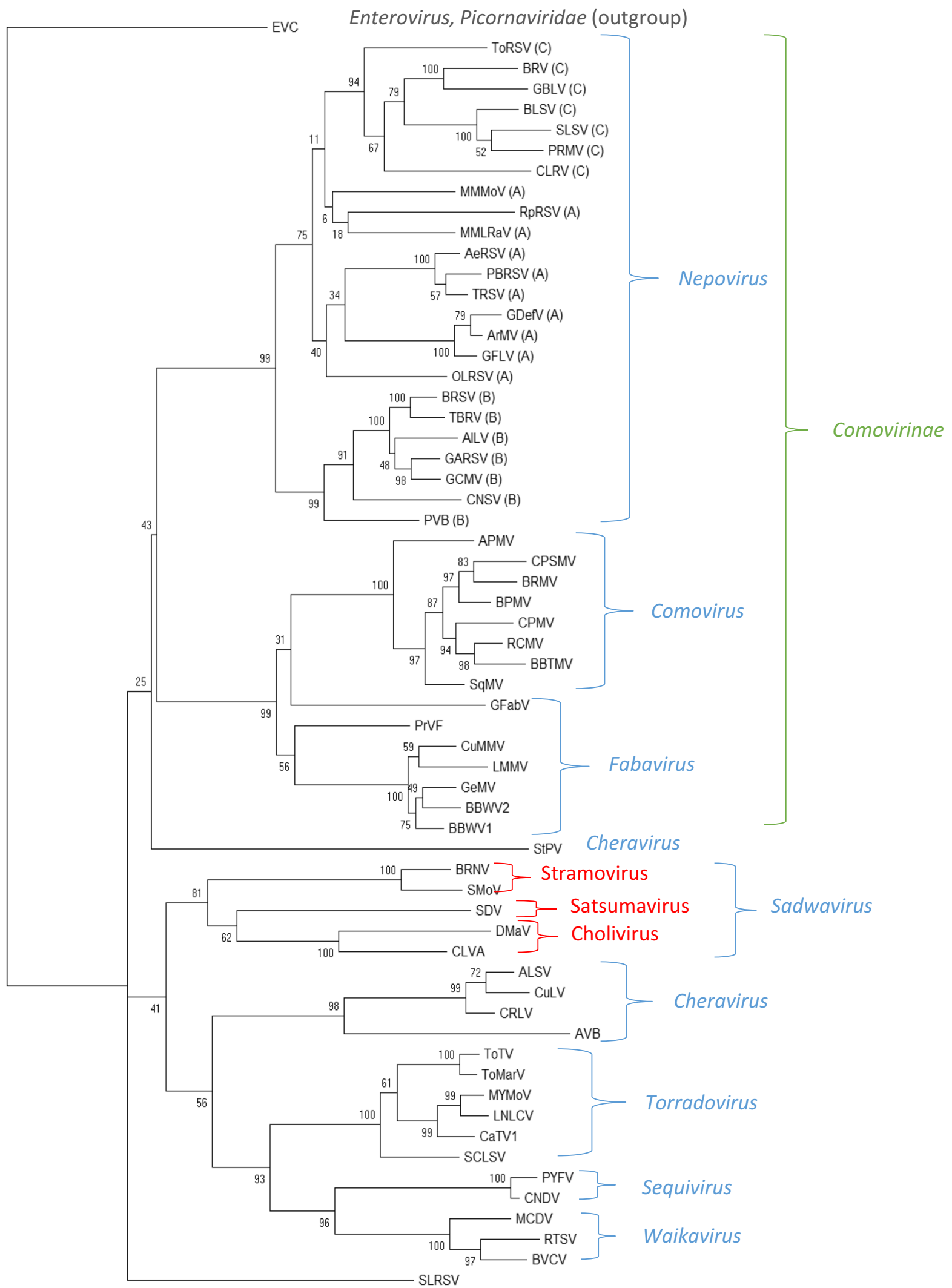


4 Fig. 3 Maximum-likelihood phylogenetic analysis of the CP amino acid sequence of type isolates from recognized species in the family Secoviridae. The alignment was generated using ClustalW in MEGA $\mathrm{X}$, and the tree was also built in MEGA X. Bootstrap values for each node are shown (1000 replicates). For each Secoviridae species, the amino acid sequence of the $\mathrm{CP}(\mathrm{s})$ was deduced from the nucleotide sequence of the corresponding genomic RNA from the type isolate. The sequences for the single CP (nepoviruses, some sadwaviruses) or the combined sequences for the two or three CPs were used for the alignment. Characterized or putative cleavage sites were used to define the borders of the CP domain(s). Unless specified, virus abbreviations are as listed in Fig. 1. Sequence accession numbers are as follows: ToRSV (D12477), BRV (AF020051), GBLV (FN691935), BLSV (AB649297), SLSV (KX424572), PRMV (KJ572573), CLRV (FR851462), MMMoV (AB518486), RpRSV (AY303788), MMLRaV (KC904084), AeRSV (JQ670669), PBRSV (KC832892), TRSV (AY363727), GDeF (AY291208), ArMV (AY017339), GFLV (X16907), OLRSV (olive latent ringspot virus, AJ277435), BRSV (X04062), TBRV (AY157994), AILV (LT608396), GARSV (AY291207), GCMV (X15163), CNSV (AB073148), PVB (KX656671), APMV (Andean potato mottle virus, L16239), CPSMV (M83309), BRMV (KP404603), BPMV (U70866), CPMV (X00729), RCMV (M14913), BBTMV (GU810904), SqMV (AB054689), GFabV (KX241485), PrVF (KX269871), CuMMV (EU881937), LMMV (KC595305), GeMV (AB084453), BBWV2 (AF225954), BBWV1 (AB084451), StPV (DQ143875), BRNV (DQ344640), SMoV (AJ311876), SDV (AB009959), DMaV (KU215539), CLVA (JN052074), ALSV (AB030941), CuLV (KT692953), CRLV (AJ621358), AVB (JQ581051), ToTV (DQ388880), ToMarV (EF681765), MYMoV (KM229701), LNLCV (KC855267), CaTV1 (KF533720), SCLSV (KU052531), PYFV (D14066), CNDV (EU980442), MCDV (U67839), RTSV (M95497), BVCV (KT238881), SLRSV (AY860979). The combined sequence of the three CPs from poliovirus (EVC, species Enterovirus C, NP_041277, genus Enterovirus, family Picornaviridae) was used as an outgroup to root the tree. Higher taxa are indicated with the following code: the three proposed subgenera in red, the genera in blue, and the subfamily in green. In the case of nepoviruses, the letter in parentheses after each virus abbreviation indicates the subgroup

\section{References}

1. Adams MJ, Lefkowitz EJ, King AM, Harrach B, Harrison RL, Knowles NJ, Kropinski AM, Krupovic M, Kuhn JH, Mushegian AR, Nibert M, Sabanadzovic S, Sanfacon H, Siddell SG, Simmonds P, Varsani A, Zerbini FM, Gorbalenya AE, Davison AJ (2016) Ratification vote on taxonomic proposals to the International Committee on Taxonomy of Viruses (2016). Arch Virol 161:2921-2949

2. Adams MJ, Lefkowitz EJ, King AMQ, Harrach B, Harrison RL, Knowles NJ, Kropinski AM, Krupovic M, Kuhn JH, Mushegian AR, Nibert M, Sabanadzovic S, Sanfacon H, Siddell SG, Simmonds P, Varsani A, Zerbini FM, Gorbalenya AE, Davison AJ (2017) Changes to taxonomy and the International Code of Virus Classification and Nomenclature ratified by the International Committee on Taxonomy of Viruses (2017). Arch Virol 162:2505-2538

3. Bhagwat B, Dickison V, Ding X, Walker M, Bernardy M, Bouthillier M, Creelman A, DeYoung R, Li Y, Nie X, Wang A, Xiang Y, Sanfacon $\mathrm{H}$ (2016) Genome sequence analysis of five Canadian isolates of strawberry mottle virus reveals extensive intra-species diversity and a longer RNA2 with increased coding capacity compared to a previously characterized European isolate. Arch Virol 161:1657-1663

4. Fuchs M, Schmitt-Keichinger C, Sanfacon H (2017) A renaissance in nepovirus research provides new insights into yheir molecular interface with hosts and vectors. Adv Virus Res 97:61-105
5. Halgren A, Tzanetakis IE, Martin RR (2007) Identification, characterization and detection of black raspberry necrosis virus. Phytopathology 97:44-50

6. Hayashi EA, Blawid R, de Melo FL, Andrade MS, Pio-Ribeiro G, de Andrade GP, Nagata T (2017) Complete genome sequence of a putative new secovirus infecting yam (Dioscorea) plants. Arch Virol 162:317-319

7. Le Gall O, Sanfacon H, Ikegami M, Iwanami T, Jones T, Karasev A, Lehto K, Wellink J, Wetzel T, Yoshikawa N (2007) Cheravirus and Sadwavirus: two unassigned genera of plant positive-sense single-stranded RNA viruses formerly considered atypical members of the genus Nepovirus (family Comoviridae). Arch Virol 159:1767-1774

8. Le Gall O, Christian P, Fauquet CM, King AM, Knowles NJ, Nakashima N, Stanway G, Gorbalenya AE (2008) Picornavirales, a proposed order of positive-sense single-stranded RNA viruses with a pseudo- $\mathrm{T}=3$ virion architecture. Arch Virol 153:715-727

9. Mann KS, Walker M, Sanfacon H (2017) Identification of cleavage sites recognized by the $3 \mathrm{C}$-like cysteine protease within the two polyproteins of strawberry mottle virus. Front Microbiol 8:745

10. Mann KS, Walker MC, Sanfacon H (2017) Corrigendum: Identification of cleavage sites recognized by the 3C-like cysteine protease within the two polyproteins of strawberry mottle virus. Front Microbiol 8:1762

11. Mann KS, Chisholm J, Sanfacon H (2019) Strawberry mottle virus (family Secoviridae, order Picornavirales) encodes a novel glutamic protease to process the RNA2 polyprotein at two cleavage sites. J Virol. https://doi.org/10.1128/JVI.01679-18

12. Mann KS, Sanfacon H (2019) Expanding repertoire of plant positive-strand RNA virus proteases. Viruses 11:66

13. Sanfacon H, Wellink J, Le Gall O, Karasev A, van der Vlugt R, Wetzel T (2009) Secoviridae: a proposed family of plant viruses within the order Picornavirales that combines the families Sequiviridae and Comoviridae, the unassigned genera Cheravirus and Sadwavirus, and the proposed genus Torradovirus. Arch Virol 154:899-907

14. Siddell SG, Walker PJ, Lefkowitz EJ, Mushegian AR, Adams MJ, Dutilh BE, Gorbalenya AE, Harrach B, Harrison RL, Junglen S, Knowles NJ, Kropinski AM, Krupovic M, Kuhn JH, Nibert M, Rubino L, Sabanadzovic S, Sanfacon H, Simmonds P, Varsani A, Zerbini FM, Davison AJ (2019) Additional changes to taxonomy ratified in a special vote by the International Committee on Taxonomy of Viruses (October 2018). Arch Virol 164:943-946

15. Thompson JR, Leone G, Lindner JL, Jelkmann W, Schoen CD (2002) Characterization and complete nucleotide sequence of strawberry mottle virus: a tentative member of a new family of bipartite plant picorna-like viruses. J Gen Virol 83:229-239

16. Thompson JR, Dasgupta I, Fuchs M, Iwanami T, Karasev AV, Petrzik K, Sanfacon H, Tzanetakis I, van der Vlugt R, Wetzel T, Yoshikawa N, ICTV_Report_Consortium (2017) ICTV virus taxonomy profile: Secoviridae. J Gen Virol 98:529-531

17. Wang A, Sanfacon H (2000) Proteolytic processing at a novel cleavage site in the $\mathrm{N}$-terminal region of the tomato ringspot nepovirus RNA-1-encoded polyprotein in vitro. J Gen Virol $81: 2771-2781$

18. Wetzel T, Chisholm J, Bassler A, Sanfacon H (2008) Characterization of proteinase cleavage sites in the $\mathrm{N}$-terminal region of the RNA1-encoded polyprotein from Arabis mosaic virus (subgroup A nepovirus). Virology 375:159-169

19. Wylie SJ, Luo H, Li H, Jones MG (2012) Multiple polyadenylated RNA viruses detected in pooled cultivated and wild plant samples. Arch Virol 157:271-284

Publisher's Note Springer Nature remains neutral with regard to jurisdictional claims in published maps and institutional affiliations. 